J3eA, Journal sur l'enseignement des sciences et technologies de l'information et des systèmes, Volume 4, Hors-Série 4, 12 (2005)

DOI : http://dx.doi.org/10.1051/bib-j3ea:2005862

(C) EDP Sciences, 2005

\title{
L'analyse de l'intensité du courant statorique : outil de surveillance et de diagnostic
}

C. Pachaud 


\title{
COMMUNICATION DANS LES JOURNEES DU CLUB EEA
}

\author{
« Diagnostic en Electronique, Electrotechnique et Automatique »
}

\author{
17-18 Mars 2005 Lyon
}

\section{L'ANALYSE DE L'INTENSITE DU COURANT STATORIQUE : OUTIL DE SURVEILLANCE ET DE DIAGNOSTIC}

\section{Christian PACHAUD}

L'analyse vibratoire est une technique d'investigation non intrusive de plus en plus utilisée dans l'industrie dans le cadre de la maintenance des machines tournantes. Cependant, le champ d'investigation offert par cette technique ne couvre pas la totalité des défauts susceptibles d'affecter une machine tournante. II en est ainsi pour les défauts induisant des fluctuations de couple et de vitesse de rotation instantanée (jeux angulaires, points durs, défauts de boucle de régulation...) qui, dans le domaine vibratoire, se manifestent essentiellement par des phénomènes de modulation d'amplitude ou de fréquence de certaines composantes cinématiques de la machine avec un apport d'énergie vibratoire très faible. Par contre, les relations beaucoup plus directes liant les variations instantanées de l'intensité du courant statorique avec celles du couple et de la vitesse de rotation font de l'analyse de cette grandeur, une technique d'investigation non intrusive très performante comme l'illustrent les deux exemples qui suivent.

Ce premier exemple concerne le groupe de commande de la première section de presses d'une machine à papier. Ce groupe est constitué d'un moteur asynchrone alimenté par un convertisseur de fréquence qui entraîne la presse inférieure par l'intermédiaire d'un réducteur et d'une transmission par cardan.

Le spectre de l'intensité du courant statorique montre une très nette modulation de sa composante principale $(37,77 \mathrm{~Hz})$ par l'ordre 2 de la fréquence de rotation de la presse inférieure. La fréquence de modulation principale $6,73 \mathrm{~Hz}$ est induite par la rotation du cardan (figure 01 ).

L'analyse de la fonction de modulation d'amplitude montre que le taux de modulation temporel est de $26,5 \%$ ce qui correspond à une variation de l'intensité du courant statorique de 45,2 A. Le taux de modulation correspondant à la fréquence de modulation principale $(6,73 \mathrm{~Hz})$ est de $20,4 \%$ (figure 02 ). Ces valeurs peuvent être considérées comme extrêmement élevées.

L'analyse de la fonction de modulation de fréquence montre que le taux de modulation temporel est de $4,6 \%$ ce qui correspond à une variation de la fréquence instantanée du courant statorique de $3,2 \mathrm{~Hz}$. Le taux de modulation correspondant à la fréquence de défaut de cardan $(6,73 \mathrm{~Hz})$ est de $2,9 \%$ (figure 03$)$. Ces valeurs sont elles aussi à considérer comme très élevées. 
Les fluctuations de couple importantes induites par le cardan ne sont pas identifiables par l'analyse vibratoire. L'examen du cardan après démontage ne révèle qu'un léger marquage des cannelures. Par contre, les effets de son remplacement se traduisent par une forte diminution des taux de modulation d'amplitude et de fréquence de l'intensité du courant statorique (figure 04) et, par voie de conséquence, des fluctuations de couple. Ces dernières se manifestaient par des variations de vitesse de rotation instantanée et un glissement des feutres très préjudiciables au niveau du procédé (accélération du processus de dégradation du feutre et des revêtements des presses par facettage, variation du grammage du papier).

Le deuxième exemple concerne un groupe hydroélectrique bulbe, constitué d'une turbine Kaplan entraînant une génératrice asynchrone à 6 pôles par l'intermédiaire d'un multiplicateur.

Après avoir constaté depuis plusieurs années un pompage très marqué de l'intensité du courant statorique par la fréquence de passage des pales de la turbine, on note l'apparition dans le spectre de ce dernier de deux familles de bandes latérales de modulation, l'une espacée de la fréquence de passage des pôles, l'autre de la fréquence de rotation de l'arbre PV (figures 05 et 06). L'apparition de ces deux phénomènes de modulation laisse présager, d'une part la présence de fissures au niveau des barres rotoriques, d'autre part l'apparition d'un jeu angulaire au niveau de la ligne PV traduisant soit une usure de l'accouplement du multiplicateur avec la turbine soit une usure de la rainure de clavette de fixation de la roue PV du multiplicateur. Les diagrammes ci-dessous donnent l'évolution des taux de modulation d'amplitude associés à chaque fréquence de modulation.

\section{Evolution du taux de modulation de l'intensité du} courant à $2 \mathrm{Fg}$

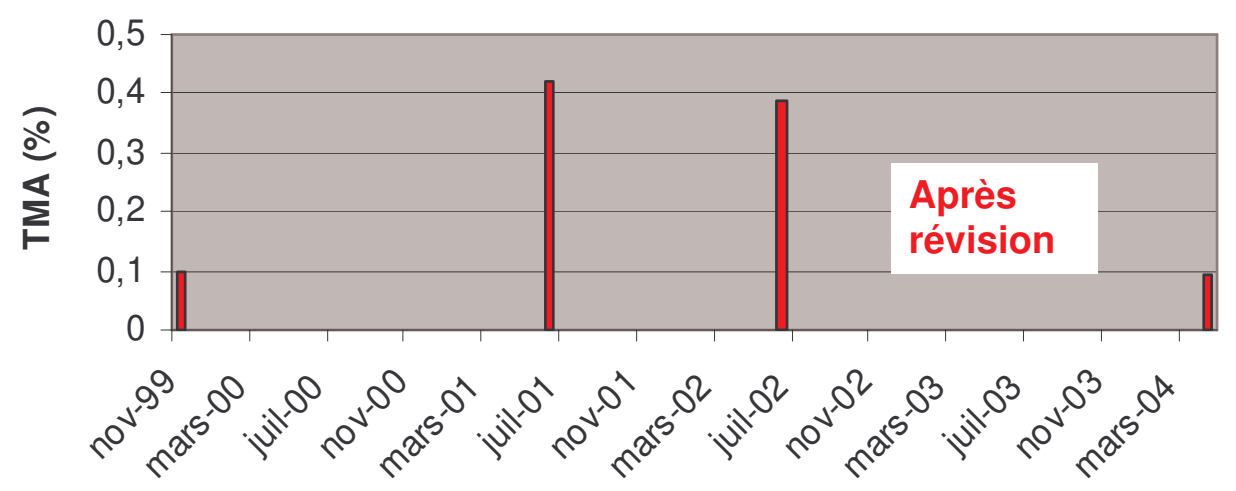


Journées EEA Section Electrotechnique - 17-18 mars 2005, Lyon

Evolution des taux de modulation de l'intensité du courant

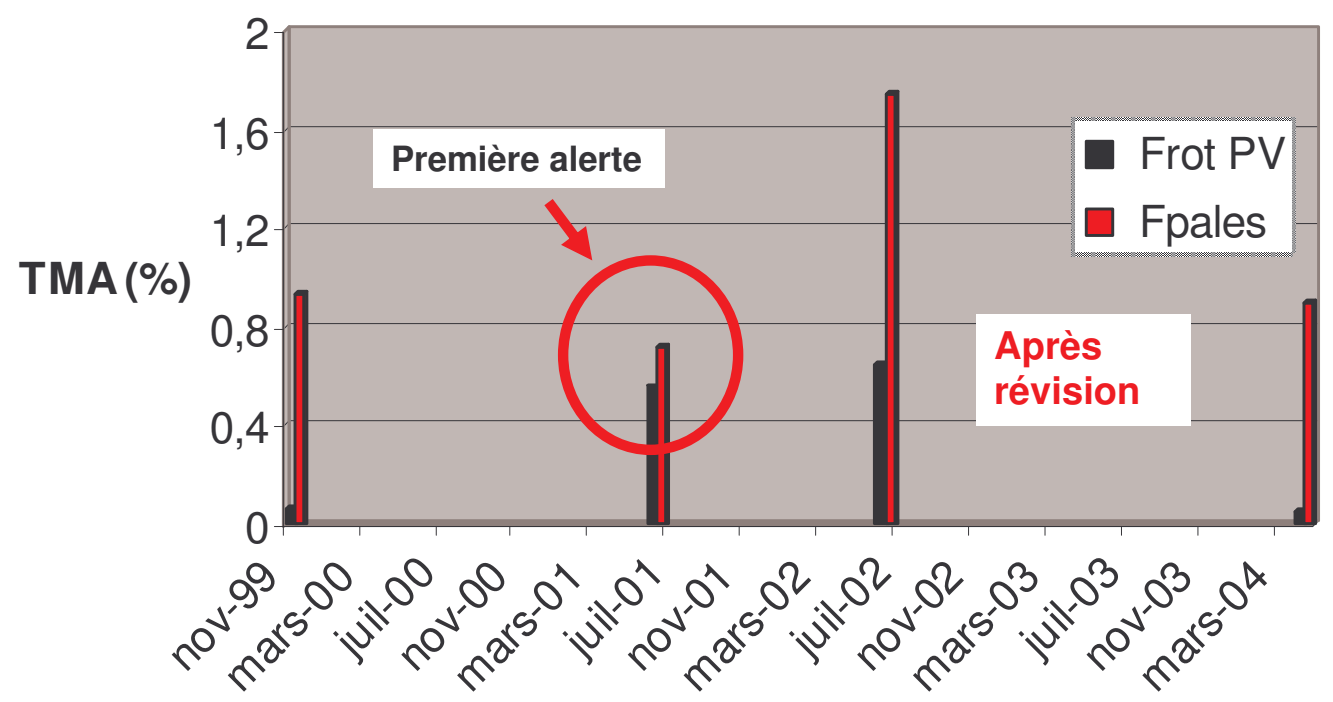


Les analyses de vibrations ne donnent pas d'informations très significatives. L'amplitude de la composante d'ordre 1 de la fréquence de rotation est très faible et aucune famille de bandes latérales de modulation n'est identifiable. On notre seulement la présence peu marquée de bandes latérales de modulation espacées de la fréquence de passage des pôles autour des fréquences d'encoches. Les niveaux vibratoires relevés sur les paliers du multiplicateur sont très faibles. Le défaut se manifeste essentiellement en direction axiale par la présence de nombreuses paires de modulation de faibles amplitudes autour de la fréquence d'engrènement du train PV/MV espacées de la fréquence de rotation de l'arbre PV qui disparaîtront après remise en état du multiplicateur (figure 07).

Le constat de démontage effectué quelques mois plus tard confirme le diagnostic. Le rotor de la génératrice présente effectivement coté accouplement deux fissures diamétralement opposées (figures 08 et 09). La rainure de clavette de fixation de la roue PV du multiplicateur est profondément fissurée (figure 10).

Ces deux exemples montrent clairement la nette performance de l'analyse de l'intensité du courant statorique sur l'analyse vibratoire traditionnelle en présence de défauts d'origine électromagnétique ou mécanique induisant des fluctuations de couple. Malheureusement, cette technique d'investigation dont les fondements remontent à plus de 20 ans, a beaucoup de difficultés à s'implanter dans le milieu industriel tout au moins en France.

Bien qu'il n'existe à ce jour aucun outil spécialement conçu à cet effet traitant directement et automatiquement les fonctions de modulation de l'intensité du courant statorique, les outils actuels de surveillance de machines offrent sans la moindre difficulté la possibilité d'un suivi d'évolution des spectres de l'intensité du courant statorique. Si les informations fournies sont parfois insuffisantes pour permettre la formulation d'un diagnostic précis, elles permettent néanmoins avec une excellente fiabilité, la détection de l'apparition de toute anomalie. La prise de mesures n'offre pas de difficultés particulières et peut, avec quelques aménagements particuliers dans le cas où le transport de l'énergie électrique s'effectue par câbles de phase non séparés, être confiée à un personnel non électricien habilité.

Le principal obstacle à la pénétration dans l'industrie de cette technique d'investigation n'est donc pas matériel mais essentiellement culturel et se situe à deux niveaux :

- la non-maîtrise du traitement du signal même sous ses formes les plus élémentaires dans beaucoup de Services Electrique, Instrumentation et Automatisme.

En effet, l'analyse spectrale du courant et les techniques de traitement du signal appliquées à cette grandeur sont loin d'être d'un usage courant dans ces Services et l'instrumentation dont ils disposent, n'offre que rarement les fonctionnalités nécessaires.

- le cloisonnement le plus souvent quasi hermétique des Services Mécanique, Electrique, Instrumentation et Automatisme.

Le Service Electrique ne se préoccupe que rarement des interactions mécanique/électrique induites par les récepteurs en aval du moteur ou par le 
procédé. De même, le service mécanique, à qui est le plus souvent confié la surveillance des machines qui nécessite la mise en œuvre des techniques de traitement du signal beaucoup plus avancées et facilement transposables à l'analyse des courants statoriques, se désintéresse totalement des aspects électriques.

Dynae, société de service spécialisée dans la surveillance et le diagnostic de machines tournantes, intègre depuis de nombreuses années dans le cadre de ses prestations de diagnostic, l'analyse du courant statorique. Par assurer la promotion de cette technique d'investigation, nous avons créé un stage industriel spécifique d'une durée de 2 jours au cours desquels sont exposés les techniques de traitement du signal dédiées à l'analyse des courants statoriques et des signaux délivrés par les capteurs de vitesse angulaire, la mise en oeuvre de ces techniques d'investigation, le tout illustré par plus de 20 cas réels. Cette action est complétée dans différentes écoles d'ingénieurs sous forme de conférences de sensibilisation.

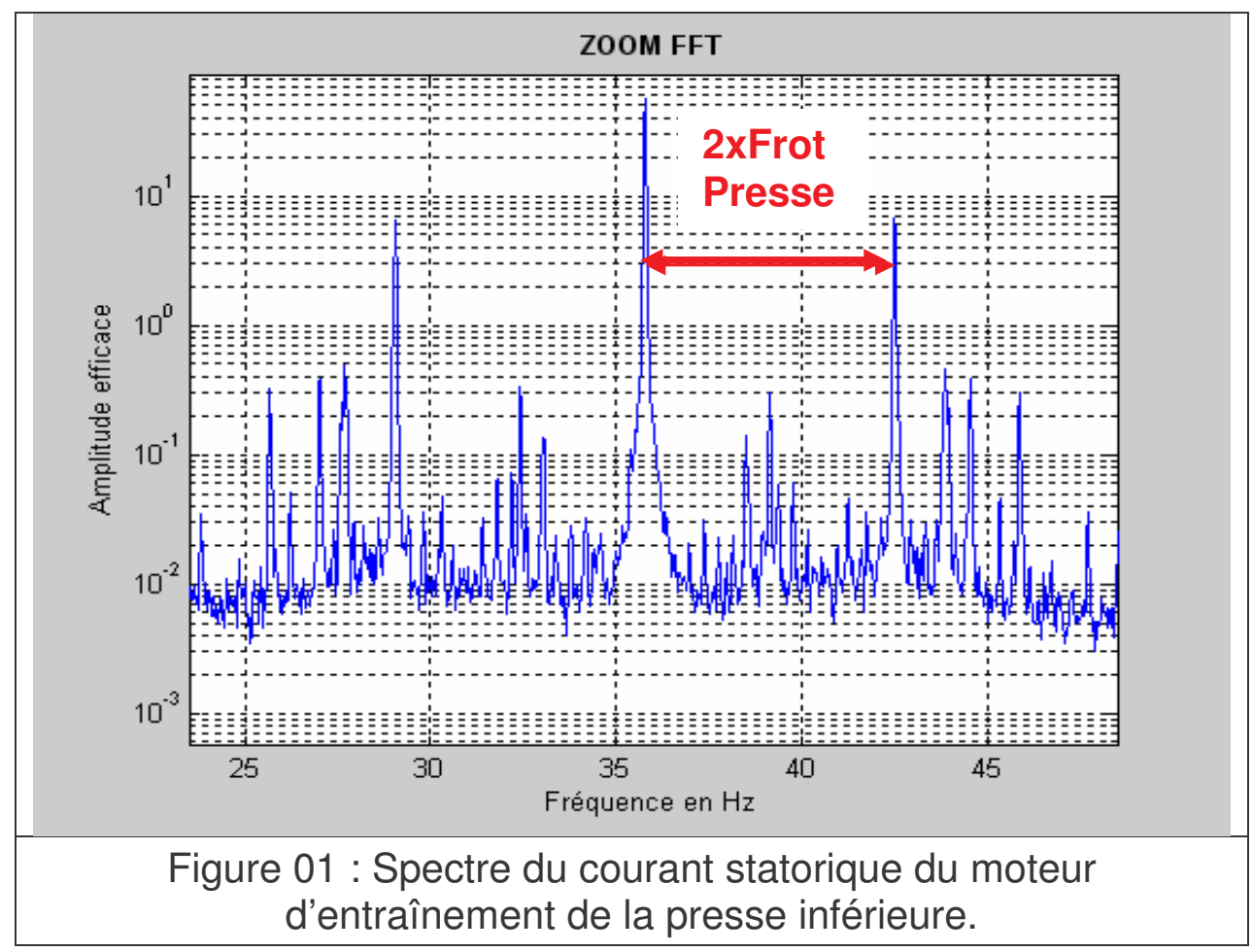



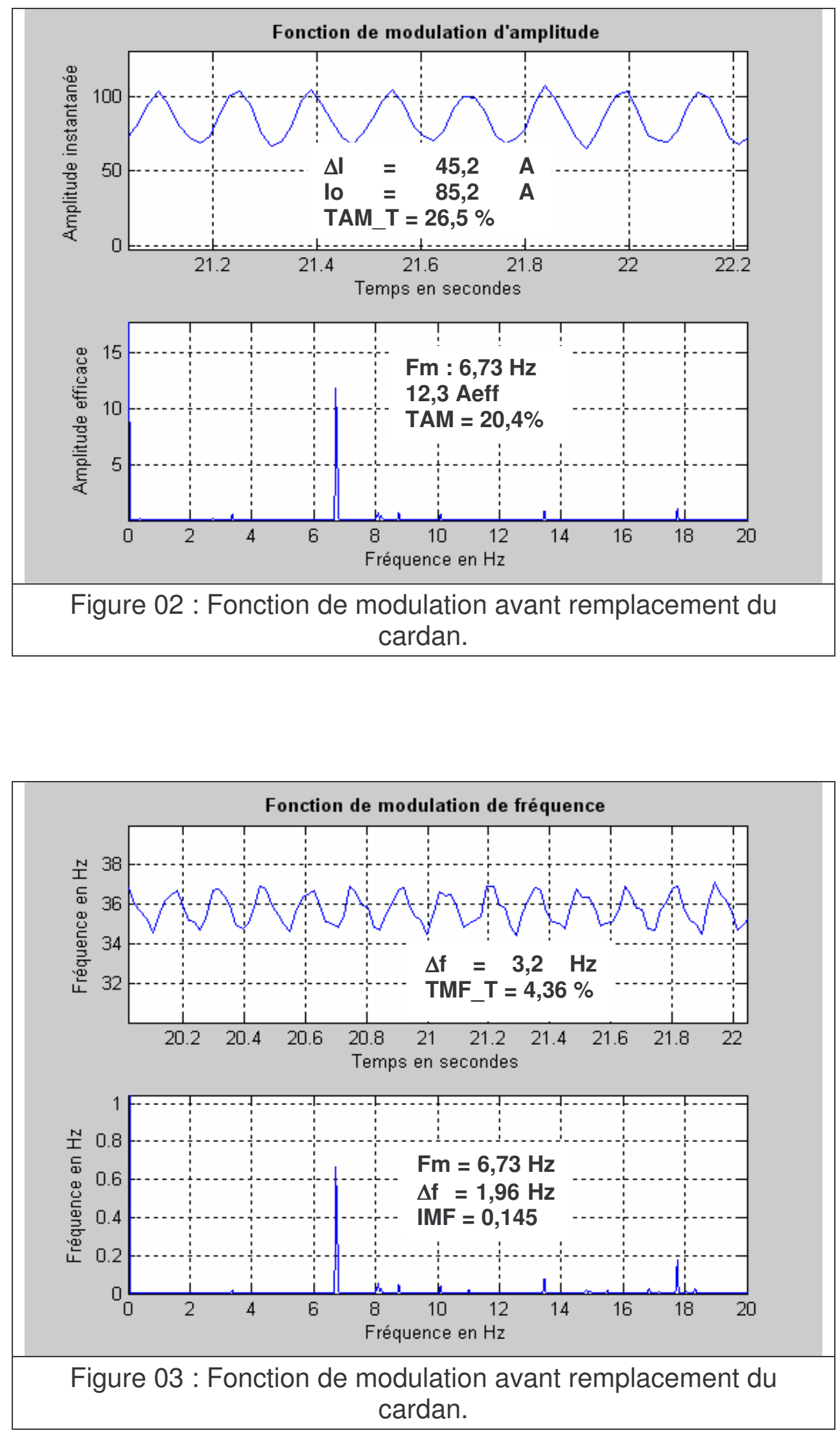

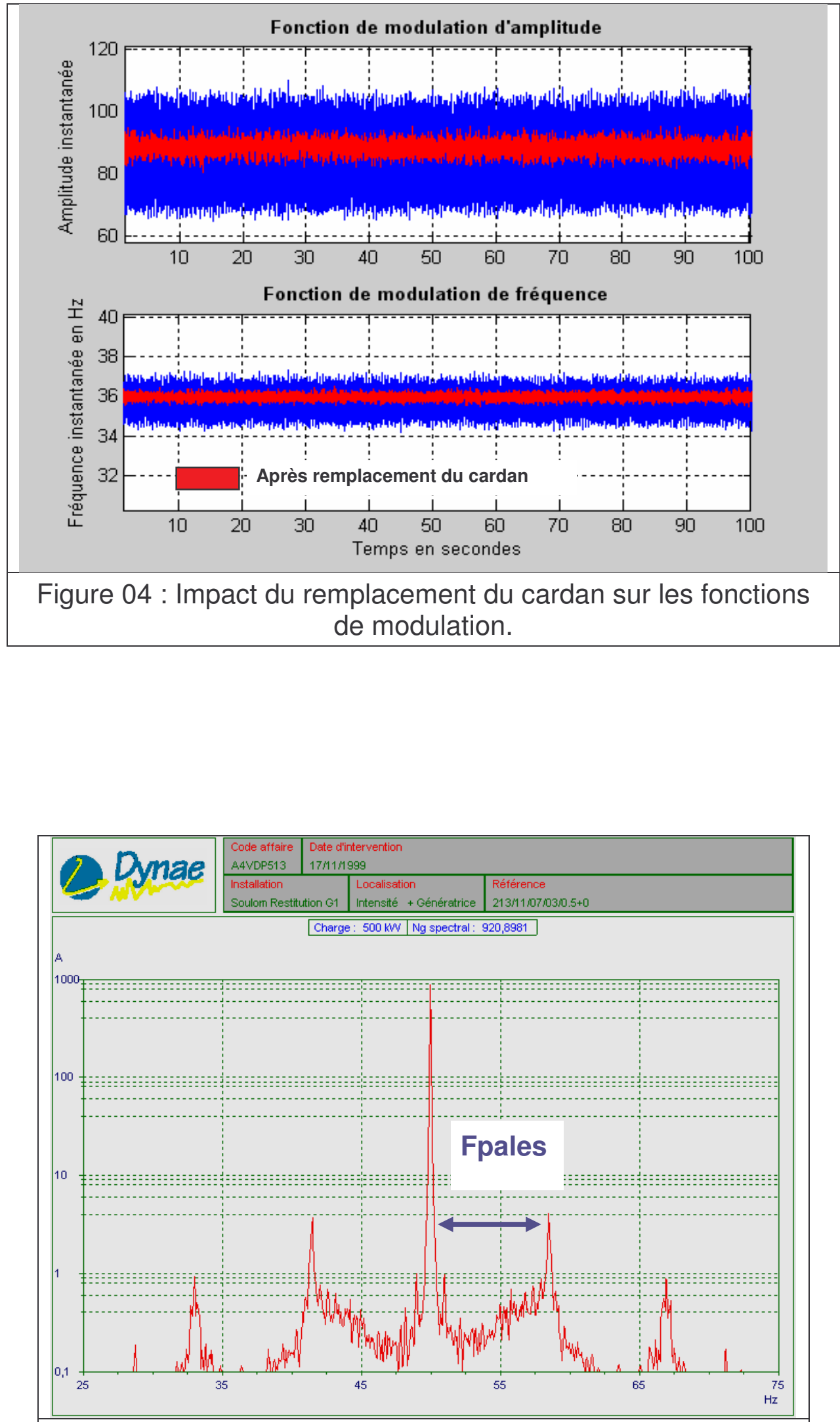

Figure 05 : Spectre du courant statorique de la génératrice. 
Journées EEA Section Electrotechnique - 17-18 mars 2005, Lyon

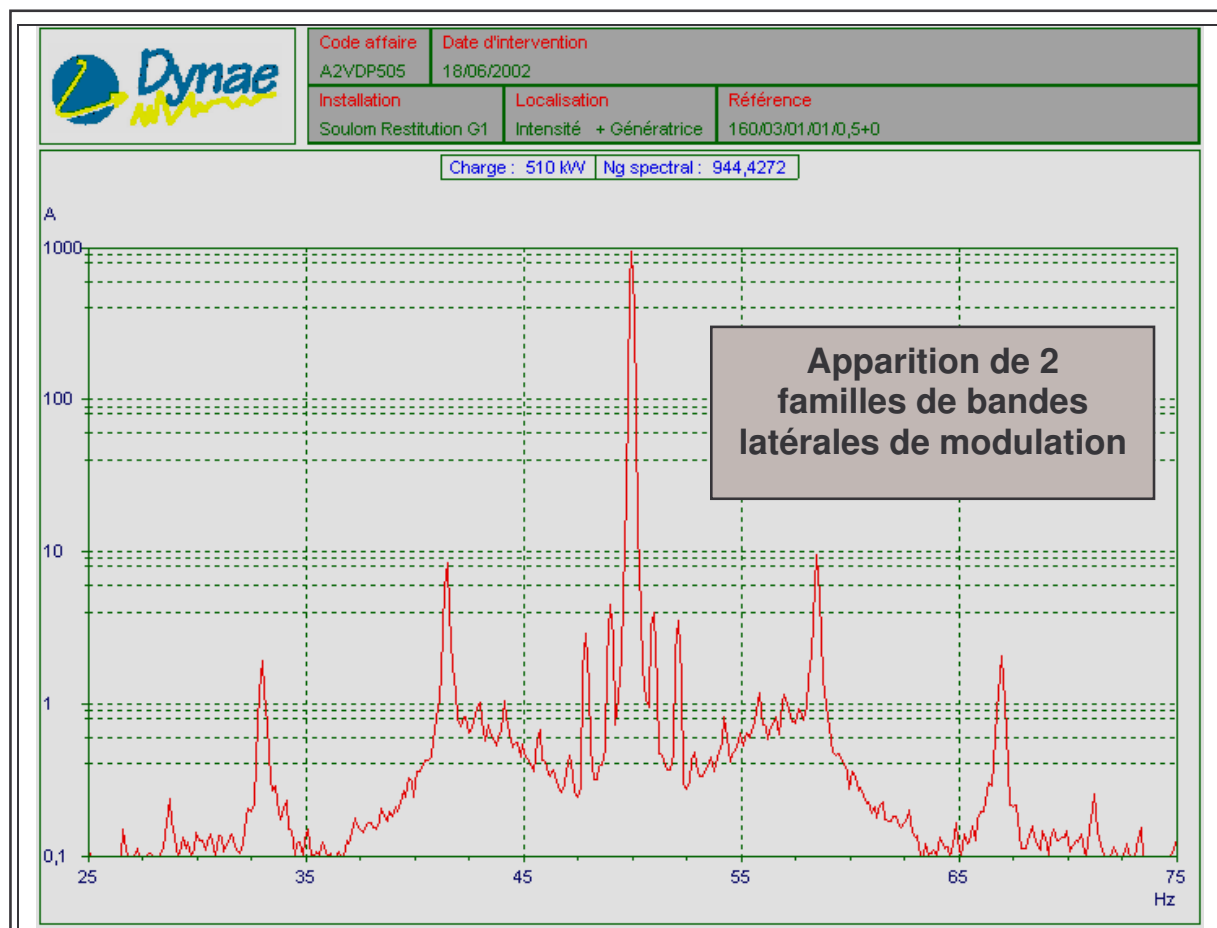

Figure 06 : Spectre du courant statorique 18 mois après. 
Journées EEA Section Electrotechnique - 17-18 mars 2005, Lyon

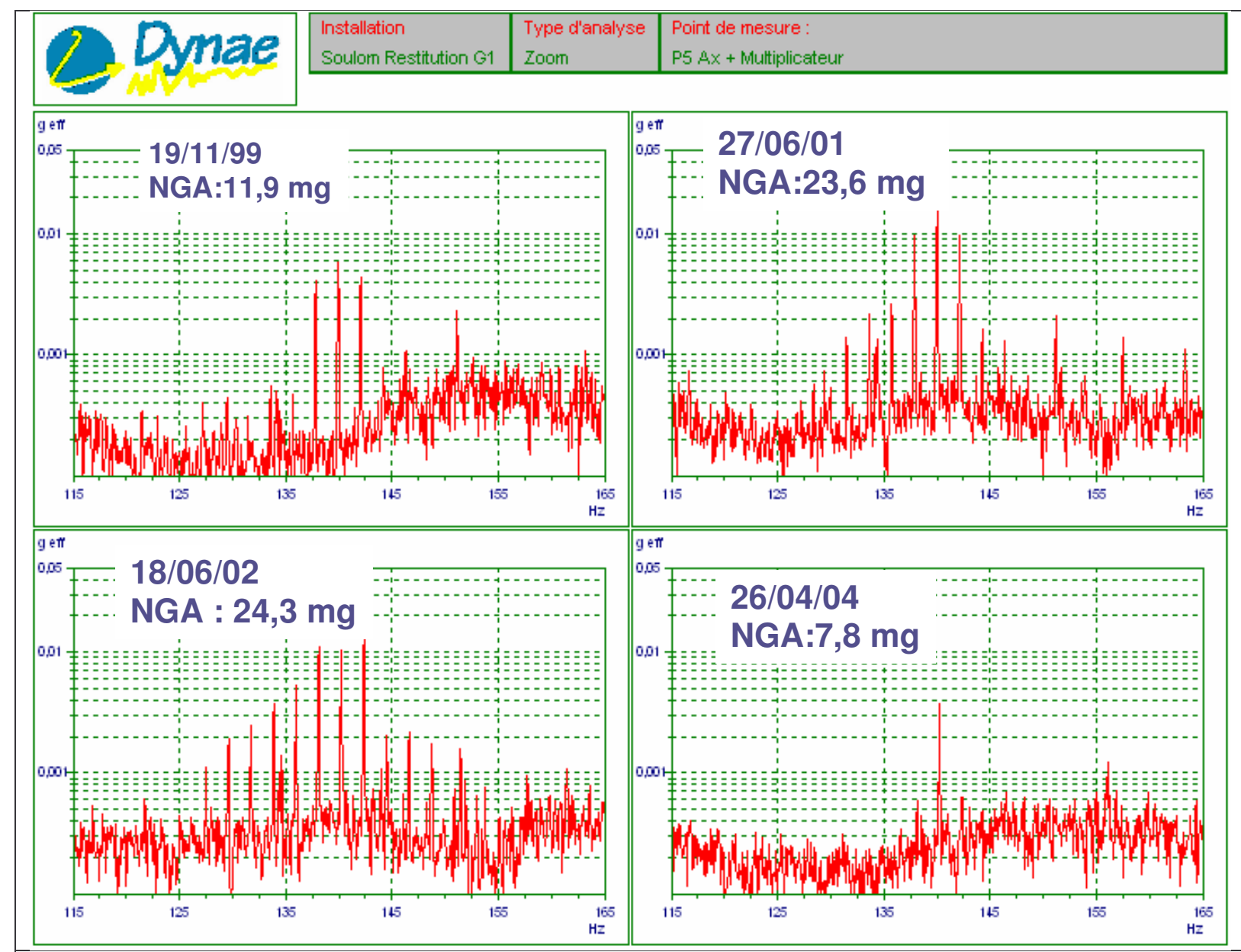

Figure 07 : Spectres vibratoires de l'engrènement PV/MV

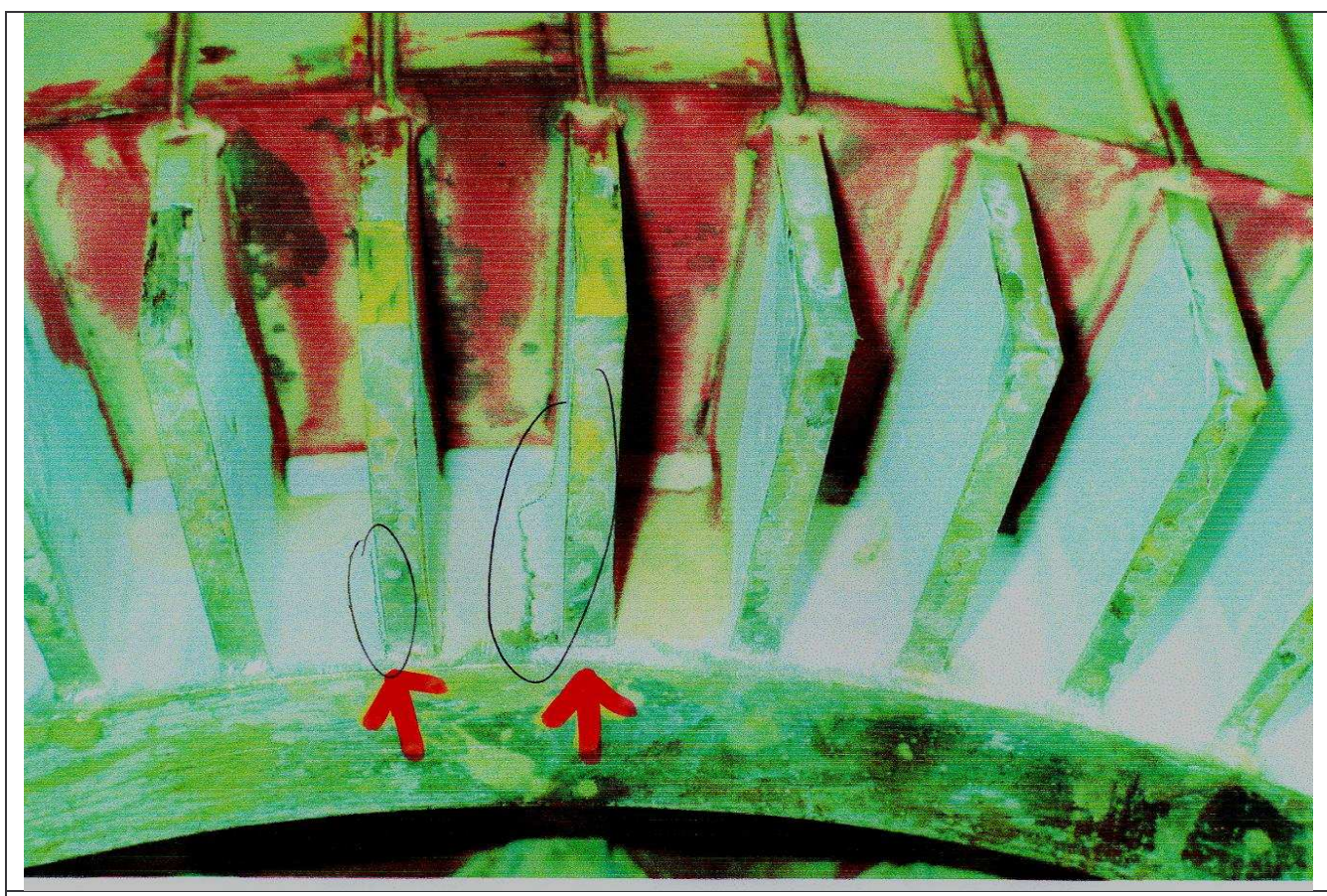

Figure 08 : Fissuration au niveau de deux liaisons barres-anneaux 
Journées EEA Section Electrotechnique - 17-18 mars 2005, Lyon

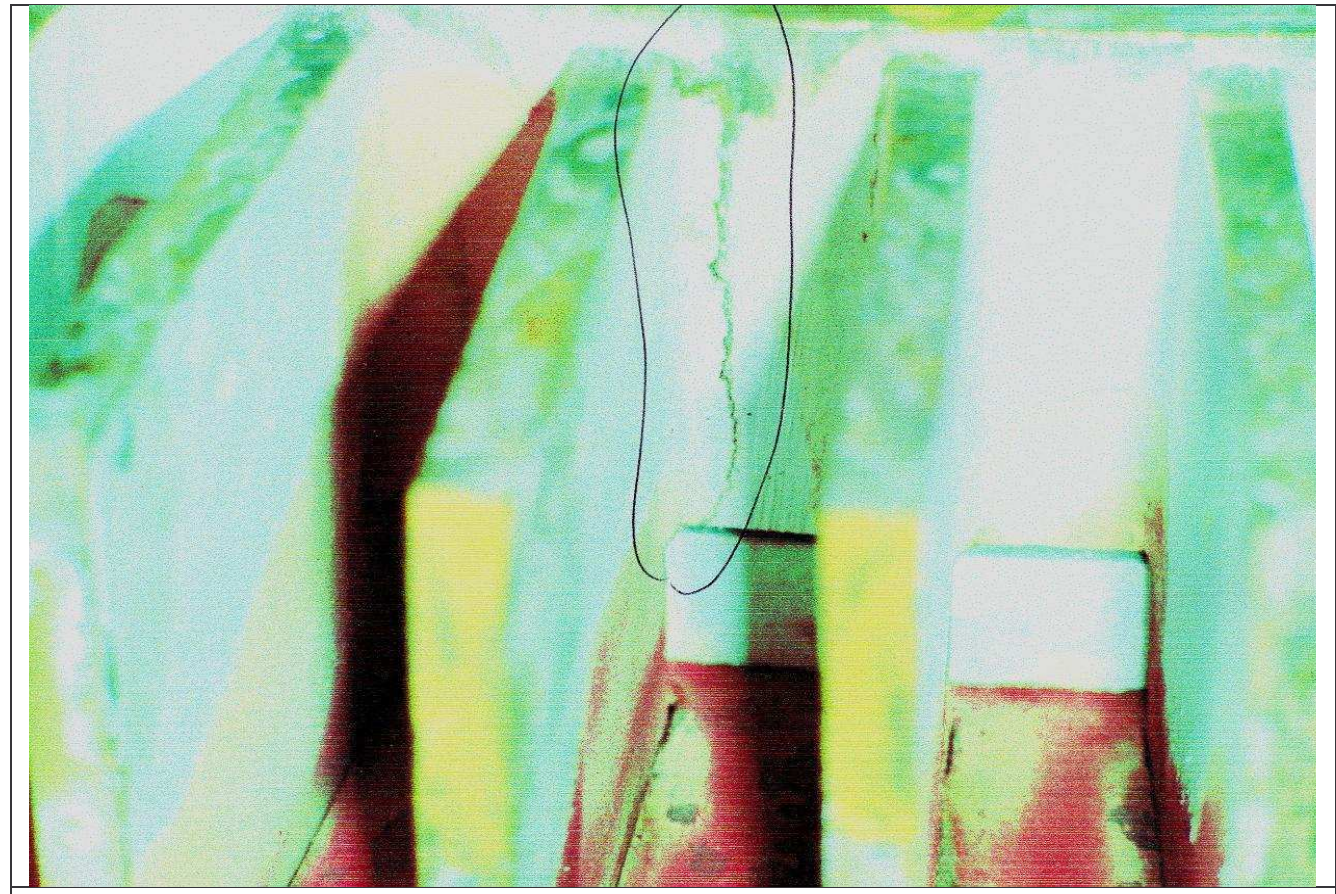

Figure 09 : Fissure diamétralement opposée aux deux précédentes

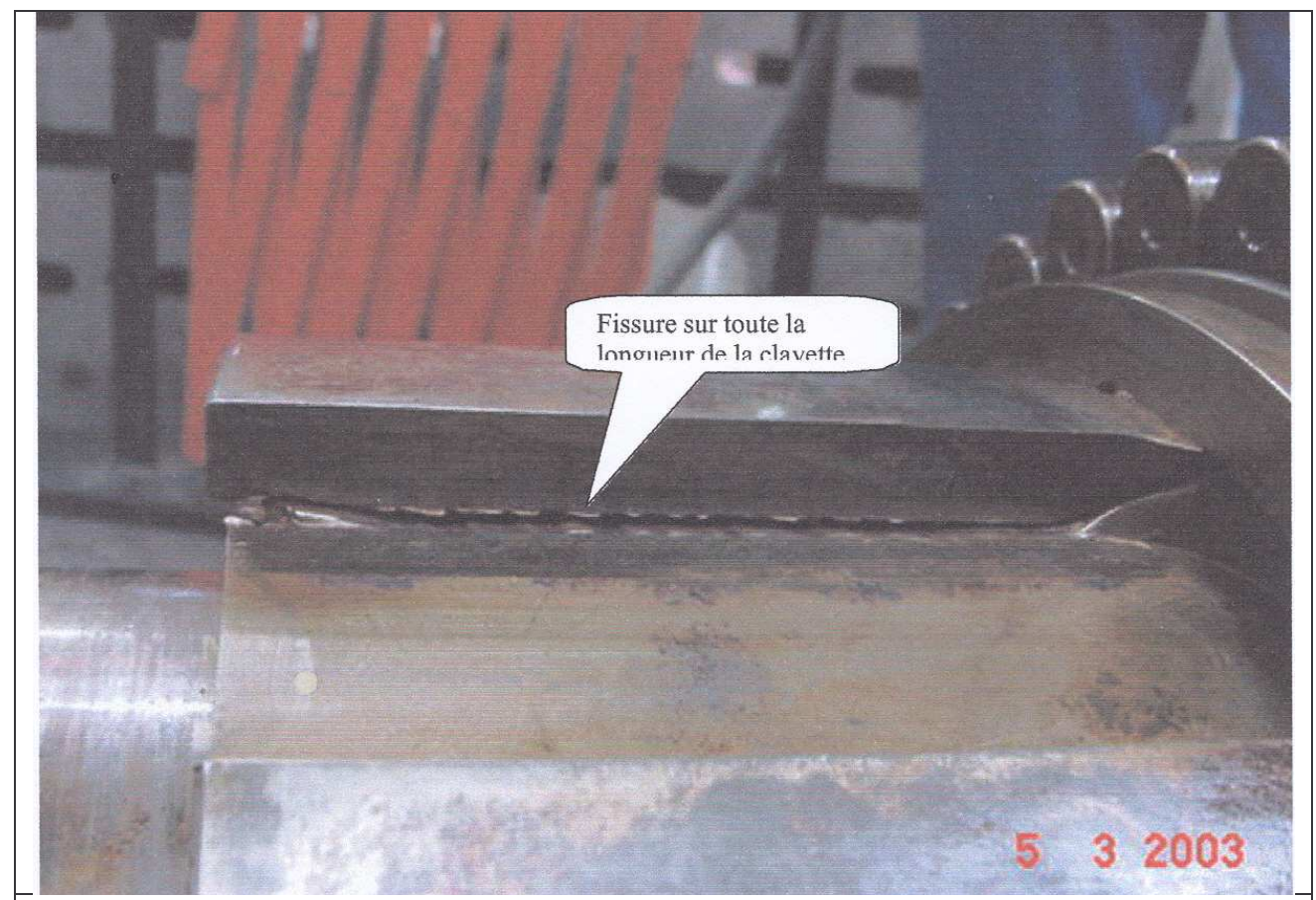

Figure 10 : Fissure de la rainure de clavette de fixation de la roue PV 
Christian PACHAUD est support technique de la société Dynae (groupe CLEMESSY /DALKIA) et responsable de son agence Sud Ouest. Dynae, société spécialisée en analyse vibratoire et surveillance multitechnique d'équipements industriels, est issue de la fusion de la société Campagna \& IND et du département MDV de la société AIF Services (maintenant NORISKO).

Docteur es Sciences, son implication professionnelle dans le domaine de l'Acoustique et des Vibrations commence en 1974 lorsqu'il crée au sein de la société AIF Services un département Acoustique Architecturale et Industrielle dans lequel les techniques vibratoires vont rapidement occuper une place importante. II crée ensuite en 1984 au sein de cette même société, le département Maintenance Diagnostic Vibration spécialisé dans le diagnostic et la surveillance de machines tournantes dont il sera le responsable technique jusqu'à la fusion de cette entité avec la société Campagna \& IND. Auteur de plusieurs publications de niveau international dédiées à l'application des techniques d'investigation vibratoires et de traitement du signal à la surveillance et au diagnostic des machines tournantes ("Contribution du facteur de crête et du kurtosis à l'identification des défauts induisant des forces impulsionnelles périodiques » Mécanique Industrielle et Matériaux (Juin 1997), "Crest factor and kurtosis contributions to identify defects inducing periodical impulsive forces », Mechanical Systems and Signal Processing (1997)). 